\title{
Genome-wide variations of SARS-CoV-2 infer evolution relationship and transmission route
}

Lehai Zhang ${ }^{1,2 \sharp}$, Shifu Wang ${ }^{1 \#}$, Qian Ren ${ }^{1}$, Junjie Yang ${ }^{3}$, Yanqin $\mathrm{Lu}^{4}$, Lei Zhang ${ }^{5,6, *}$, Zhongtao Gai ${ }^{2, *}$

1. Department of Children's Medical Laboratory Diagnosis Center, Qilu Children's Hospital of Shandong University, Jinan 250022, China

2. Jinan Institute of Pediatrics \& Shandong Children's Microbiome Center, Qilu Children's Hospital of Shandong University, Jinan, Shandong, 250022, China

3. College of Life Science, Qilu Normal University, Jinan, Shandong, 250200, China

4. Key Laboratory for Biotech-Drugs of National Health Commission, Key Laboratory for Rare \& Uncommon Diseases of Shandong Province, Shandong Medicinal Biotechnology Centre, Shandong First Medical University \& Shandong Academy of Medical Sciences, Jinan 250062, China

5. Microbiome-X, Beijing Advanced Innovation Center for Big Data-Based Precision Medicine, School of Medicine and Engineering, Beihang University, \& Key Laboratory of Big Data-Based Precision Medicine (Beihang University), the Ministry of Industry and Information Technology of the People's Republic of China, Beijing, 100191, China

6. Shandong Institute of Industrial Technology for Health Sciences and Precision Medicine, Jinan, Shandong, 250101, China

The authors have withdrawn this manuscript (Genome-wide variations of SARS-CoV-2 infer evolution relationship and transmission route) from medRxiv, because it was found that the statistical and analytical methods used in the manuscript had certain controversies after further discussion, so the authors of the manuscipt disclaimed that this conclusion cannot be used as the basis for the origin and evolution of SARS-COV-2, also as information to guide clinical practice and health-related behaviors, it should not be reported as established facts in 
medRxiv preprint doi: https://doi.org/10.1101/2020.04.27.20081349; this version posted May 8, 2020. The copyright holder for this preprint (which was not certified by peer review) is the author/funder, who has granted medRxiv a license to display the preprint in perpetuity.

All rights reserved. No reuse allowed without permission.

the news media and "We-Media". No one should do too much extended interpretation of the content of this manuscript. Therefore, the authors do not wish this work to be cited as reference for the project. If you have any questions, please contact the corresponding author. 